Honam Mathematical J. 35 (2013), No. 4, pp. 667-677

http://dx.doi.org/10.5831/HMJ.2013.35.4.667

\title{
CERTAIN UNIFIED INTEGRALS INVOLVING A PRODUCT OF BESSEL FUNCTIONS OF THE FIRST KIND
}

\author{
Junesang Choi* and Praveen Agarwal
}

\begin{abstract}
A remarkably large number of integrals involving a product of certain combinations of Bessel functions of several kinds as well as Bessel functions, themselves, have been investigated by many authors. Motivated the works of both Garg and Mittal and Ali, very recently, Choi and Agarwal gave two interesting unified integrals involving the Bessel function of the first kind $J_{\nu}(\mathrm{z})$. In the present sequel to the aforementioned investigations and some of the earlier works listed in the reference, we present two generalized integral formulas involving a product of Bessel functions of the first kind, which are expressed in terms of the generalized Lauricella series due to Srivastava and Daoust. Some interesting special cases and (potential) usefulness of our main results are also considered and remarked, respectively.
\end{abstract}

\section{Introduction and preliminaries}

The study of Bessel functions has a very long history (see, e.g., [10]) and now stands on fairly firm footing through the research contributions of various authors (see, e.g., $[2,10,11,20,21]$ and [22]). Bessel functions are important special functions and their closely related ones are widely used in physics and engineering; therefore, they are of interest to physicists and engineers as well as mathematicians. So it looks natural that

Received August 16, 2013. Accepted September 11, 2013.

2010 Mathematics Subject Classification. Primary 33B20, 33C20; Secondary 33B15, 33C05.

Key words and phrases. Gamma function, Hypergeometric function ${ }_{2} F_{1}$, generalized hypergeometric function ${ }_{p} F_{q}$, generalized (wright) hypergeometric functions ${ }_{p} \Psi_{q}$, generalized hypergeometric series, generalized Lauricella series in several variables, cosine and sine trigonometric functions, Bessel function of the first kind, Oberhettinger's integral formula.

${ }^{*}$ Corresponding author 
many research works on the further investigations of the Bessel functions have recently come up. Numerous integral formulas involving a variety of special functions have been developed by many authors (see, e.g., [5]; for a very recent work, see also [7]). Also many integral formulas associated with the Bessel functions of several kinds have been presented (see, e.g., [5, 196-204]; see also [21, 373-476]). Those integrals involving Bessel functions are not only of great interest to the pure mathematics, but they are often of extreme importance in many branches of theoretical and applied physics and engineering. Several methods for evaluating infinite integrals involving Bessel functions have been known (see, e.g., [1] and [12]). However, these methods usually work on a case-by-case basis.

Motivated the works of both Garg and Mittal [9] and Ali [3], very recently, Choi and Agarwal [6] gave two interesting unified integrals involving the Bessel function of the first kind $J_{\nu}(\mathrm{z})(1.1)$. In the present sequel to the aforementioned investigations, we present two generalized integral formulas involving a product of Bessel functions of the first kind, which are expressed in terms of the generalized Lauricella series (1.2) due to Srivastava and Daoust [17]. Some interesting special cases and (potential) usefulness of our main results are also considered and remarked, respectively.

For our purpose, we begin by recalling some known functions and earlier works. The Bessel function of the first kind $J_{\nu}(z)$ is defined for $z \in \mathbb{C} \backslash\{0\}$ and $\nu \in \mathbb{C}$ with $\Re(\nu)>-1$ by the following series (see, e.g., [12, p. 217, Entry 10.2.2] and [21, p. 40, Eq. (8)]):

$$
J_{\nu}(z)=\sum_{k=0}^{\infty} \frac{(-1)^{k}\left(\frac{z}{2}\right)^{\nu+2 k}}{k ! \Gamma(\nu+k+1)},
$$

where $\mathbb{C}$ denotes the set of complex numbers and $\Gamma(z)$ is the familiar Gamma function (see, e.g., [16, Section 1.1]).

An interesting further several-variable-generalization of the generalized Lauricella series (see, e.g., [19, p. 36, Eq. (19)]) is defined by (cf. Srivastava and Daoust [17, p. 454]; see also [19, p. 37]) 


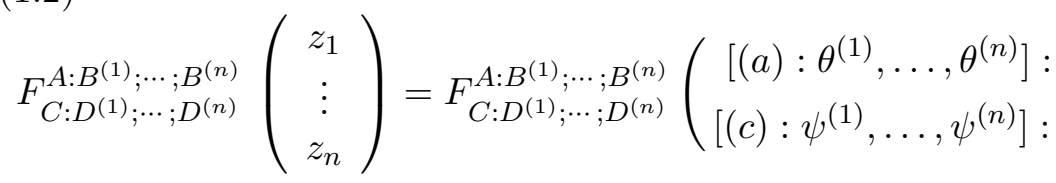

$$
\begin{aligned}
& \begin{array}{l}
{\left[(b)^{(1)}: \phi^{(1)}\right] ; \ldots ;\left[(b)^{(n)}: \phi^{(n)}\right] ;} \\
{\left[(d)^{(1)}: \delta^{(1)}\right] ; \ldots ;\left[(d)^{(n)}: \delta^{(n)}\right] ;}
\end{array} \\
& =\sum_{k_{1}, \ldots, k_{n}=0}^{\infty} \Omega\left(k_{1}, \ldots, k_{n}\right) \frac{z_{1}^{k_{1}}}{k_{1} !} \cdots \frac{z_{n}^{k_{n}}}{k_{n} !},
\end{aligned}
$$

where, for convenience,

$$
\Omega\left(k_{1}, \ldots, k_{n}\right)=\frac{\prod_{j=1}^{A}\left(a_{j}\right)_{k_{1} \theta_{j}^{(1)}+\cdots+k_{n} \theta_{j}^{(n)}} \prod_{j=1}^{B^{(1)}}\left(b_{j}^{(1)}\right)_{k_{1} \phi_{j}^{(1)}} \cdots \prod_{j=1}^{B^{(n)}}\left(b_{j}^{(n)}\right)_{k_{n} \phi_{j}^{(n)}}}{\prod_{j=1}^{C}\left(c_{j}\right)_{k_{1} \psi_{j}^{(1)}+\cdots+k_{n} \psi_{j}^{(n)}} \prod_{j=1}^{D^{(1)}}\left(d_{j}^{(1)}\right)_{k_{1} \delta_{j}^{(1)}} \cdots \prod_{j=1}^{D^{(n)}}\left(d_{j}^{(n)}\right)_{k_{n} \delta_{j}^{(n)}}},
$$

the coefficients

$$
\left\{\begin{array}{l}
\theta_{j}^{(m)}(j=1, \ldots, A) ; \phi_{j}^{(m)}\left(j=1, \ldots, B^{(m)}\right) ; \\
\psi_{j}^{(m)}(j=1, \ldots, C) ; \delta_{j}^{(m)}\left(j=1, \ldots, D^{(m)}\right) ; \forall m \in\{1, \ldots, n\}
\end{array}\right.
$$

are real and positive, and $(a)$ abbreviates the array of $A$ parameters $a_{1}, \ldots, a_{A},\left(b^{(m)}\right)$ abbreviates the array of $B^{(m)}$ parameters

$$
b_{j}^{(m)} \quad\left(j=1, \ldots, B^{(m)}\right) ; \quad \forall m \in\{1, \ldots, n\},
$$

with similar interpretations for $(c)$ and $\left(d^{(m)}\right)(m=1, \ldots, n)$; et cetera.

The multiple series (1.2) converges absolutely either

(i) $\Delta_{i}>0(i=1, \ldots, n), \forall z_{1}, \cdots, z_{n} \in \mathbb{C}$,

or

(ii) $\Delta_{i}=0(i=1, \ldots, n), \forall z_{1}, \cdots, z_{n} \in \mathbb{C},\left|z_{i}\right|<\varrho_{i}(i=1, \ldots, n)$.

The multiple series (1.2) is divergent when $\Delta_{i}<0(i=1, \cdots, n)$ except for the trivial case $z_{1}=0, \ldots, z_{n}=0$. Here

$$
\Delta_{i} \equiv 1+\sum_{j=1}^{C} \psi_{j}^{(i)}+\sum_{j=1}^{D^{(i)}} \delta_{j}^{(i)}-\sum_{j=1}^{A} \theta_{j}^{(i)}-\sum_{j=1}^{B^{(i)}} \phi_{j}^{(i)}(i=1, \ldots, n)
$$


and

$$
\varrho_{i}=\min _{\mu_{1}, \ldots, \mu_{n}>0}\left\{E_{i}\right\} \quad(i=1, \cdots, n)
$$

with

$$
E_{i}=\left(\mu_{i}\right) 1+\sum_{j=1}^{D^{(i)}} \delta_{j}^{(i)}-\sum_{j=1}^{B^{(i)}} \phi_{j}^{(i)} \frac{\left\{\prod_{j=1}^{C}\left(\sum_{i=1}^{n} \mu_{i} \psi_{j}^{(i)}\right)^{\psi_{j}^{(i)}}\right\}\left\{\prod_{j=1}^{D^{(i)}}\left(\delta_{j}^{(i)}\right)^{\delta_{j}^{(i)}}\right\}}{\left\{\prod_{j=1}^{A}\left(\sum_{i=1}^{n} \mu_{i} \theta_{j}^{(i)}\right)^{\theta_{j}^{(i)}}\right\}\left\{\prod_{j=1}^{B^{(i)}}\left(\phi_{j}^{(i)}\right)^{\phi_{j}^{(i)}}\right\}} .
$$

For more details, the reader may be referred (for example) to the earlier work by Srivastava and Daoust [17]. Special cases of (1.2) have been established in terms of generalized hypergeometric functions of one and two variables, respectively (see, e.g., [19, pp. 39-40]). For the sake of completeness, we recall the familiar generalized hypergeometric series ${ }_{p} F_{q}$ defined by (see, e.g., [16, Section 1.5])

$$
\begin{aligned}
{ }_{p} F_{q}\left[\begin{array}{c}
\alpha_{1}, \ldots, \alpha_{p} ; z \\
\beta_{1}, \ldots, \beta_{q} ; z
\end{array}\right] & =\sum_{n=0}^{\infty} \frac{\left(\alpha_{1}\right)_{n} \cdots\left(\alpha_{p}\right)_{n}}{\left(\beta_{1}\right)_{n} \cdots\left(\beta_{q}\right)_{n}} \frac{z^{n}}{n !} \\
& ={ }_{p} F_{q}\left(\alpha_{1}, \ldots, \alpha_{p} ; \beta_{1}, \ldots, \beta_{q} ; z\right),
\end{aligned}
$$

where $(\lambda)_{n}$ is the Pochhammer symbol defined (for $\lambda \in \mathbb{C}$ ) by (see [16, p. 2 and pp. 4-6]):

$$
\begin{aligned}
(\lambda)_{n}: & =\left\{\begin{array}{lc}
1 & (n=0) \\
\lambda(\lambda+1) \ldots(\lambda+n-1) & (n \in \mathbb{N}:=\{1,2,3, \ldots\})
\end{array}\right. \\
& =\frac{\Gamma(\lambda+n)}{\Gamma(\lambda)} \quad\left(\lambda \in \mathbb{C} \backslash \mathbb{Z}_{0}^{-}\right)
\end{aligned}
$$

and $\mathbb{Z}_{0}^{-}$denotes the set of nonpositive integers.

We also need to recall the following Oberhettinger's integral formula [13]:

$$
\int_{0}^{\infty} x^{\mu-1}\left(x+a+\sqrt{x^{2}+2 a x}\right)^{-\lambda} d x=2 \lambda a^{-\lambda}\left(\frac{a}{2}\right)^{\mu} \frac{\Gamma(2 \mu) \Gamma(\lambda-\mu)}{\Gamma(1+\lambda+\mu)},
$$

provided $0<\Re(\mu)<\Re(\lambda)$.

\section{Main results}

We establish two generalized integral formulas whose integrands are a finite product of Bessel functions of the first kind (1.1) multiplied by the integrand of (1.10). The resulting integrals are found to be expressed in terms of the 
generalized Lauricella functions (1.2) as given in Theorem 1 and Theorem 2 below.

Theorem 1. The following integral formula holds true: For $\lambda, \mu, \nu_{j} \in \mathbb{C}$ with $\Re\left(\nu_{j}\right)>-1,0<\Re(\mu)<\Re\left(\lambda+\nu_{j}\right)(j=1, \ldots, n)$ and $x>0$,

$$
\begin{aligned}
\int_{0}^{\infty} x^{\mu-1}\left(x+a+\sqrt{x^{2}+2 a x}\right)^{-\lambda} \prod_{j=1}^{n} J_{\nu_{j}}\left(\frac{y_{j}}{x+a+\sqrt{x^{2}+2 a x}}\right) d x \\
=2^{1-\mu} a^{\mu-\lambda}\left(\prod_{j=1}^{n} \frac{\left(\frac{y_{j}}{2 a}\right)^{\nu_{j}}}{\Gamma\left(\nu_{j}+1\right)}\right) \frac{\Gamma(2 \mu) \Gamma\left(1+\lambda+\sum_{j=1}^{n} \nu_{j}\right) \Gamma\left(\lambda-\mu+\sum_{j=1}^{n} \nu_{j}\right)}{\Gamma\left(\lambda+\sum_{j=1}^{n} \nu_{j}\right) \Gamma\left(1+\lambda+\mu+\sum_{j=1}^{n} \nu_{j}\right)} \\
\cdot F_{2: 1 ; \ldots ; 1}^{2: 0 ; \ldots ; 0}\left[\begin{array}{l}
{\left[1+\lambda+\sum_{j=1}^{n} \nu_{j}: 2, \ldots, 2\right],\left[\lambda-\mu+\sum_{j=1}^{n} \nu_{j}: 2, \ldots, 2\right]:} \\
{\left[1+\lambda+\mu+\sum_{j=1}^{n} \nu_{j}: 2, \ldots, 2\right],\left[\lambda+\sum_{j=1}^{n} \nu_{j}: 2, \ldots, 2\right]:} \\
\left.\left[\nu_{1}+1: 1\right] ; \ldots ;\left[\nu_{n}+1: 1\right] ;-\frac{y_{1}^{2}}{4 a^{2}}, \ldots,-\frac{y_{n}^{2}}{4 a^{2}}\right] .
\end{array}\right.
\end{aligned}
$$

Theorem 2. The following integral formula holds true: For $\lambda, \mu, \nu_{j} \in \mathbb{C}$ with $\Re\left(\nu_{j}\right)>-1,0<\Re(\mu)<\Re\left(\lambda+\nu_{j}\right)(j=1, \cdots, n)$ and $x>0$,

$$
\begin{array}{r}
\int_{0}^{\infty} x^{\mu-1}\left(x+a+\sqrt{x^{2}+2 a x}\right)^{-\lambda} \prod_{j=1}^{n} J_{\nu_{j}}\left(\frac{x y_{j}}{x+a+\sqrt{x^{2}+2 a x}}\right) d x \\
=2^{1-\mu} a^{\mu-\lambda}\left(\prod_{j=1}^{n} \frac{\left(\frac{y_{j}}{4}\right)^{\nu_{j}}}{\Gamma\left(\nu_{j}+1\right)}\right) \frac{\Gamma(\lambda-\mu) \Gamma\left(1+\lambda+\sum_{j=1}^{n} \nu_{j}\right) \Gamma\left(2 \mu+2 \sum_{j=1}^{n} \nu_{j}\right)}{\Gamma\left(\lambda+\sum_{j=1}^{n} \nu_{j}\right) \Gamma\left(1+\lambda+\mu+2 \sum_{j=1}^{n} \nu_{j}\right)} \\
\cdot F_{2: 1 ; \ldots ; 1}^{2: 0 ; \ldots ; 0}\left[\begin{array}{r}
{\left[1+\lambda+\sum_{j=1}^{n} \nu_{j}: 2, \ldots, 2\right], \quad\left[2 \mu+2 \sum_{j=1}^{n} \nu_{j}: 4, \ldots, 4\right]:} \\
{\left[1+\lambda+\mu+2 \sum_{j=1}^{n} \nu_{j}: 4, \ldots, 4\right], \quad\left[\lambda+\sum_{j=1}^{n} \nu_{j}: 2, \ldots, 2\right]:} \\
\left.\left.; \nu_{1}+1: 1\right] ; \ldots ;\left[\nu_{n}+1: 1\right] ;-\frac{y_{1}^{2}}{16}, \ldots,-\frac{y_{n}^{2}}{16}\right] .
\end{array}\right.
\end{array}
$$

Proof. For convenience, let the left-hand side of the assertion (2.1) be denoted by $\mathcal{I}$. By applying (1.1) to the integrand of (2.1) and then interchanging the order of integral sign and summation, which is verified by uniform convergence of the involved series under the given conditions, we get 


$$
\begin{aligned}
\mathcal{I}= & \int_{0}^{\infty} x^{\mu-1}\left(x+a+\sqrt{x^{2}+2 a x}\right)^{-\lambda} \\
& \cdot \sum_{k_{1}=0}^{\infty}(-1)^{k_{1}} \frac{\left(\frac{y_{1}}{2\left(x+a+\sqrt{x^{2}+2 a x}\right)}\right)^{\nu_{1}+2 k_{1}}}{k_{1} ! \Gamma\left(\nu_{1}+k_{1}+1\right)} \\
& \ldots \sum_{k_{n}=0}^{\infty}(-1)_{n}^{k} \frac{\left(\frac{y_{n}}{2\left(x+a+\sqrt{x^{2}+2 a x}\right)}\right)^{\nu_{n}+2 k_{n}}}{k_{n} ! \Gamma\left(\nu_{n}+k_{n}+1\right)} d x
\end{aligned}
$$

and

$$
\begin{aligned}
\mathcal{I}= & \sum_{k_{1}, \cdots, k_{n}=0}^{\infty} \frac{(-1)^{k_{1}}\left(y_{1} / 2\right)^{\nu_{1}+2 k_{1}}}{k_{1} ! \Gamma\left(\nu_{1}+1\right)\left(\nu_{1}+1\right)_{k_{1}}} \cdots \frac{(-1)^{k_{n}}\left(y_{n} / 2\right)^{\nu_{n}+2 k_{n}}}{k_{n} ! \Gamma\left(\nu_{n}+1\right)\left(\nu_{n}+1\right)_{k_{n}}} \\
& \cdot \int_{0}^{\infty} x^{\mu-1}\left(x+a+\sqrt{x^{2}+2 a x}\right)^{-\lambda-\nu_{1}-\cdots-\nu_{n}-2 k_{1}-\cdots-2 k_{n}} d x .
\end{aligned}
$$

In view of the conditions given in Theorem 1 , since

$$
\begin{gathered}
\Re\left(\nu_{j}\right)>-1, \quad 0<\Re(\mu)<\Re\left(\lambda+\nu_{j}\right) \leq \Re\left(\lambda+\nu_{j}+2 k_{j}\right) \\
\left(k \in \mathbb{N}_{0}:=\mathbb{N} \cup\{0\} \quad \text { and } j=1, \ldots, n\right),
\end{gathered}
$$

we can apply the integral formula (1.10) to the integral in (2.3) and obtain the following expression:

$$
\begin{aligned}
\mathcal{I}=2^{1-\mu} a^{\mu-\lambda} \sum_{k_{1}, \ldots, k_{n}=0}^{\infty} \frac{(-1)^{k_{1}}\left(y_{1} / 2\right)^{\nu_{1}+2 k_{1}}}{k_{1} ! \Gamma\left(\nu_{1}+1\right)\left(\nu_{1}+1\right)_{k_{1}}} \cdots \frac{(-1)^{k_{n}}\left(y_{n} / 2\right)^{\nu_{n}+2 k_{n}}}{k_{n} ! \Gamma\left(\nu_{n}+1\right)\left(\nu_{n}+1\right)_{k_{n}}} \\
\\
\cdot \frac{\Gamma(2 \mu) \Gamma\left(\lambda-\mu+\nu_{1}+\cdots+\nu_{n}+2 k_{1}+\cdots+2 k_{n}\right)}{\Gamma\left(1+\lambda+\mu+\nu_{1}+\cdots+\nu_{n}+2 k_{1}+\cdots+2 k_{n}\right)} \\
\cdot\left(\lambda+\nu_{1}+\cdots+\nu_{n}+2 k_{1}+\cdots+2 k_{n}\right) a^{-\left(\nu_{1}+\cdots+\nu_{n}+2 k_{1}+\cdots+2 k_{n}\right)} .
\end{aligned}
$$

And we have

$$
\begin{aligned}
\mathcal{I}= & 2^{1-\mu} a^{\mu-\lambda} \sum_{k_{1}, \cdots, k_{n}=0}^{\infty} \frac{(-1)^{k_{1}}\left(y_{1} / 2\right)^{\nu_{1}+2 k_{1}}}{k_{1} ! \Gamma\left(\nu_{1}+1\right)\left(\nu_{1}+1\right)_{k_{1}}} \cdots \frac{(-1)^{k_{n}}\left(y_{n} / 2\right)^{\nu_{n}+2 k_{n}}}{k_{n} ! \Gamma\left(\nu_{n}+1\right)\left(\nu_{n}+1\right)_{k_{n}}} \\
& \cdot \frac{\Gamma 2 \mu) \Gamma\left(\lambda-\mu+\nu_{1}+\cdots+\nu_{n}+2 k_{1}+\cdots+2 k_{n}\right)}{\Gamma\left(1+\lambda+\mu+\nu_{1}+\cdots+\nu_{n}+2 k_{1}+\cdots+2 k_{n}\right)} \\
& \cdot\left(\lambda+\nu_{1}+\cdots+\nu_{n}+2 k_{1}+\cdots+2 k_{n}\right) \cdot a^{-\left(\lambda+\nu_{1}+\cdots+\nu_{n}+2 k_{1}+\cdots+2 k_{n}\right)} .
\end{aligned}
$$

Therefore we find that 
$(2.4)$

$$
\begin{aligned}
\mathcal{I}=2^{1-\mu} a^{\mu-\lambda}\left(\prod_{j=1}^{n} \frac{\left(\frac{y_{j}}{2 a}\right)^{\nu_{j}}}{\Gamma\left(\nu_{j}+1\right)}\right) \frac{\Gamma(2 \mu) \Gamma\left(\lambda-\mu+\sum_{j=1}^{n} \nu_{j}\right) \Gamma\left(1+\lambda+\sum_{j=1}^{n} \nu_{j}\right)}{\Gamma\left(1+\lambda+\mu+\sum_{j=1}^{n} \nu_{j}\right) \Gamma\left(\lambda+\sum_{j=1}^{n} \nu_{j}\right)} \\
\cdot \sum_{k_{1}, \cdots, k_{n}=0}^{\infty} \frac{\left(\lambda-\mu+\sum_{j=1}^{n} \nu_{j}\right)_{2 k_{1}+\cdots+2 k_{n}}\left(1+\lambda+\sum_{j=1}^{n} \nu_{j}\right)_{2 k_{1}+\cdots+2 k_{n}}}{\left(\lambda+\sum_{j=1}^{n} \nu_{j}\right)_{2 k_{1}+\cdots+2 k_{n}}\left(1+\lambda+\mu+\sum_{j=1}^{n} \nu_{j}\right)_{2 k_{1}+\cdots+2 k_{n}}} \\
\cdot \frac{1}{\left(\nu_{1}+1\right)_{k_{1}} \cdots\left(\nu_{n}+1\right)_{k_{n}}} \frac{\left(-y_{1}^{2} / 4 a^{2}\right)^{k_{1}}}{k_{1} !} \cdots \frac{\left(-y_{n}^{2} / 4 a^{2}\right)^{k_{n}}}{k_{n} !} .
\end{aligned}
$$

Finally, we interpret the multiple series in (2.4) as a special case of the general hypergeometric series in several variables defined by (1.2). We are thus led to the assertion (2.1). The assertion (2.2) of the Theorem 2.2 can be proved by a similar argument.

Remark 1. It is easily seen that if we set $n=1$ in (2.1) and (2.2) we can arrive at the Equations (2.1) and (2.2) in Choi and Praveen [6].

\section{Special cases}

In this section, we derive certain new integral formulas for the cosine and sine functions involving in the integrands of $(2.1)$ and (2.2). To do this, we recall the following known formula (see, e.g., [8, p. 79, Eq. (15)]):

$$
J_{-1 / 2}(z)=\sqrt{\frac{2}{\pi z}} \cos z .
$$

Setting $\nu_{1}=\cdots=\nu_{n}=-\frac{1}{2}$ in (2.1) and (2.2), and applying the expression in (3.1) to the resulting identities, we obtain two integral formulas stated in Corollary 1 and Corollary 2 below.

Corollary 1. Let the condition of Theorem 1 be satisfied. Then the following integral formula holds true:

$$
\begin{aligned}
\int_{0}^{\infty} & x^{\mu-1}\left(x+a+\sqrt{x^{2}+2 a x}\right)^{-\lambda} \\
& \cdot \prod_{j=1}^{n}\left\{\sqrt{\frac{2\left(x+a+\sqrt{x^{2}+2 a x}\right)}{\pi y_{j}}} \cos \left(\frac{y_{j}}{x+a+\sqrt{x^{2}+2 a x}}\right)\right\} d x \\
= & 2^{1-\mu+\frac{n}{2}} \pi^{-\frac{n}{2}} a^{\mu-\lambda+\frac{n}{2}}\left(\prod_{j=1}^{n} \frac{1}{\sqrt{y_{j}}}\right) \frac{\Gamma(2 \mu) \Gamma\left(1+\lambda-\frac{1}{2} n\right) \Gamma\left(\lambda-\mu-\frac{1}{2} n\right)}{\Gamma\left(\lambda-\frac{1}{2} n\right) \Gamma\left(1+\lambda+\mu-\frac{1}{2} n\right)}
\end{aligned}
$$




$$
\cdot F_{2: 1 ; \ldots ; 1}^{2: 0 ; \ldots ; 0}\left[\begin{array}{l}
{\left[1+\lambda-\frac{1}{2} n: 2, \ldots, 2\right],\left[\lambda-\mu-\frac{1}{2} n: 2, \ldots, 2\right]:} \\
{\left[1+\lambda+\mu-\frac{1}{2} n: 2, \ldots, 2\right],\left[\lambda-\frac{1}{2} n: 2, \ldots, 2\right]:} \\
\left.\frac{1}{[1 / 2: 1] ; \ldots ;[1 / 2: 1] ;} ;-\frac{y_{1}^{2}}{4 a^{2}}, \ldots,-\frac{y_{n}^{2}}{4 a^{2}}\right] .
\end{array}\right.
$$

Corollary 2. Let the condition of Theorem 2 be satisfied. Then the following integral formula holds true:

$$
\begin{gathered}
\int_{0}^{\infty} x^{\mu-1}\left(x+a+\sqrt{x^{2}+2 a x}\right)^{-\lambda} \\
\cdot \prod_{j=1}^{n}\left\{\sqrt{\frac{2\left(x+a+\sqrt{x^{2}+2 a x}\right)}{\pi x y_{j}}} \cos \left(\frac{x y_{j}}{x+a+\sqrt{x^{2}+2 a x}}\right)\right\} d x \\
=2^{1-\mu+n} \pi^{-\frac{n}{2}} a^{\mu-\lambda}\left(\prod_{j=1}^{n} \frac{1}{\sqrt{y_{j}}}\right) \frac{\Gamma(\lambda-\mu) \Gamma\left(1+\lambda-\frac{1}{2} n\right) \Gamma(2 \mu-n)}{\Gamma\left(\lambda-\frac{1}{2} n\right) \Gamma(1+\lambda+\mu-n)} \\
\cdot F_{2: 1 ; \ldots ; 1}^{2: 0 ; 0}\left[\begin{array}{c}
{\left[1+\lambda-\frac{1}{2} n: 2, \ldots, 2\right],[2 \mu-n: 4, \ldots, 4]:} \\
{[1+\lambda+\mu-n: 4, \ldots, 4],\left[\lambda-\frac{1}{2} n: 2, \ldots, 2\right]:} \\
\left.[1 / 2: 1] ; \ldots ;[1 / 2: 1] ;-\frac{y_{1}^{2}}{16}, \ldots,-\frac{y_{n}^{2}}{16}\right] .
\end{array}\right.
\end{gathered}
$$

Remark 2. It is easily seen that if we set $n=1$ in (3.2) and (3.3), we can arrive at the Equations (3.2) and (3.3) in Choi and Praveen [6].

We also recall the following formula (see, e.g., [8, p. 79, Eq. (14)]):

$$
J_{1 / 2}(z)=\sqrt{\frac{2}{\pi z}} \sin z .
$$

Just as in finding the formulas (3.2) and (3.3), setting $\nu_{1}=\cdots=\nu_{n}=\frac{1}{2}$ in (2.1) and (2.2), and applying the expression in (3.4) to the resulting identities, we get two integral formulas asserted in Corollary 3 and Corollary 4 below.

Corollary 3. Let the condition of Theorem 1 be satisfied. Then the following integral formula holds true:

$$
\begin{aligned}
\int_{0}^{\infty} & x^{\mu-1}\left(x+a+\sqrt{x^{2}+2 a x}\right)^{-\lambda} \\
\cdot & \prod_{j=1}^{n}\left\{\sqrt{\frac{2\left(x+a+\sqrt{x^{2}+2 a x}\right)}{\pi y_{j}}} \sin \left(\frac{y_{j}}{x+a+\sqrt{x^{2}+2 a x}}\right)\right\} d x
\end{aligned}
$$




$$
\begin{gathered}
=2^{1-\mu+\frac{n}{2}} \pi^{-\frac{n}{2}} a^{\mu-\lambda-\frac{n}{2}}\left(\prod_{j=1}^{n} \sqrt{y_{j}}\right) \frac{\Gamma(2 \mu) \Gamma\left(1+\lambda+\frac{1}{2} n\right) \Gamma\left(\lambda-\mu+\frac{1}{2} n\right)}{\Gamma\left(\lambda+\frac{1}{2} n\right) \Gamma\left(1+\lambda+\mu+\frac{1}{2} n\right)} \\
\cdot F_{2: 1 ; \ldots ; 1}^{2: 0 ; \ldots ; 0}\left[\begin{array}{l}
{\left[1+\lambda+\frac{1}{2} n: 2, \ldots, 2\right],\left[\lambda-\mu+\frac{1}{2} n: 2, \ldots, 2\right]:} \\
{\left[1+\lambda+\mu+\frac{1}{2} n: 2, \ldots, 2\right],\left[\lambda+\frac{1}{2} n: 2, \ldots, 2\right]:} \\
\left.[3 / 2: 1] ; \ldots ;[3 / 2: 1] ;-\frac{y_{1}^{2}}{4 a^{2}}, \ldots,-\frac{y_{n}^{2}}{4 a^{2}}\right] .
\end{array}\right.
\end{gathered}
$$

Corollary 4. Let the condition of Theorem 2 be satisfied. Then the following integral formula holds true:

$$
\begin{gathered}
\int_{0}^{\infty} x^{\mu-1}\left(x+a+\sqrt{x^{2}+2 a x}\right)^{-\lambda} \\
\cdot \prod_{j=1}^{n}\left\{\sqrt{\frac{2\left(x+a+\sqrt{x^{2}+2 a x}\right)}{\pi x y_{j}}} \sin \left(\frac{x y_{j}}{x+a+\sqrt{x^{2}+2 a x}}\right)\right\} d x \\
=2^{1-\mu} \pi^{-\frac{n}{2}} a^{\mu-\lambda}\left(\prod_{j=1}^{n} \sqrt{y_{j}}\right) \frac{\Gamma(\lambda-\mu) \Gamma\left(1+\lambda+\frac{1}{2} n\right) \Gamma(2 \mu+n)}{\Gamma\left(\lambda+\frac{1}{2} n\right) \Gamma(1+\lambda+\mu+n)} \\
\cdot F_{2: 1 ; \ldots ; 1}^{2: 0 ; \ldots ; 0}\left[\begin{array}{c}
{\left[1+\lambda+\frac{1}{2} n: 2, \ldots, 2\right],[2 \mu+n: 4, \ldots, 4]:} \\
{[1+\lambda+\mu+n: 4, \ldots, 4],\left[\lambda+\frac{1}{2} n: 2, \ldots, 2\right]:} \\
\left.[3 / 2: 1] ; \ldots ;[3 / 2: 1] ;-\frac{y_{1}^{2}}{16}, \ldots,-\frac{y_{n}^{2}}{16}\right] .
\end{array}\right.
\end{gathered}
$$

Remark 3. It is easily seen that if we set $n=1$ in (3.5) and (3.6), we are led to the Equations (3.7) and (3.8) in Choi and Praveen [6].

\section{Concluding remarks}

Here we briefly consider another variation of the results derived in the preceding sections. Bessel functions are important special functions that arise widely in science and engineering. Bessel functions of the first kind $J_{\nu}(z)$ are oscillatory and may be regarded as generalizations of trigonometric functions. Indeed, for large argument $z \geq 1$, the function $\sqrt{\frac{\pi z}{2}} J_{\nu}(z)$ is well approximated by the trigonometric function $\cos \left(z-\frac{\pi \nu}{2}-\frac{\pi}{4}\right)$ (see, e.g., [14, Eq. (9.3)]). Certain special cases of integrals involving a finite product of Bessel functions of the first kind $J_{\nu}(\mathrm{z})$ of the type (2.1) have been investigated in the literature by a number of authors with different arguments. For $n=3$, the infinite integral 
(2.1) with different argument was evaluated independently by Bailey [4, p. 45, Eq.(7.1)] and Rice [15, p. 60, Eq.(2.6)] who used different methods and discussed a number of interesting special cases of their main integral involving the product of three Bessel functions. In the sequel, Srivastava and Exton [18] also applied their integral formula involving the product of several Bessel functions to give an explicit expression of a generalized random walk. Therefore, the results presented in this paper are easily converted in terms of a similar type of new interesting integrals with different arguments after some suitable parametric replacements. We are also trying to find certain possible applications of those results presented here to some other research areas like random walk in [18].

\section{References}

[1] M. Abramowitz and I. A. Stegun (Editors), Handbook of Mathematical Functions with Formulas, Graphs, and Mathematical Tables, Tenth Printing, National Bureau of Standards, Applied Mathematics Series 55, National Bureau of Standards, Washington, D.C., 1972; Reprinted by Dover Publications, New York, 1965 (see also [12]).

[2] G. E. Andrews, R. Askey and R. Roy, Special Functions, Encyclopedia of Mathematics and Its Applications, Vol. 71, Cambridge University Press, Cambridge, London and New York, 1999.

[3] S. Ali, On some new unified integrals, Adv. Comput. Math. Appl. 1(3) (2012), 151-153.

[4] W. N. Bailey, Some infinite integrals involving Bessel functions, Proc. London Math. Soc. (2) 40 (1936), 37-48.

[5] Y. A. Brychkov, Handbook of Special Functions, Derivatives, Integrals, Series and Other Formulas, CRC Press, Taylor \& Francis Group, Boca Raton, London, and New York, 2008.

[6] J. Choi and P. Agarwal, Certain unified integrals associated with Bessel functions, Boundary Value Problems 2013, 2013:95.

[7] J. Choi, A. Hasanov, H. M. Srivastava and M. Turaev, Integral representations for Srivastava's triple hypergeometric functions, Taiwanese J. Math. 15 (2011), 2751-2762.

[8] A. Erdélyi, W. Magnus, F. Oberhettinger, and F. G. Tricomi, Higher Transcendental Functions, Vol. II, McGraw-Hill Book Company, New York, Toronto, and London, 1953.

[9] M. Garg and S. Mittal, On a new unified integral, Proc. Indian Acad. Sci. (Math. Sci.) 114(2) (2003), 99-101.

[10] A. Gray and G. B. Mathews, A Treatise on Bessel functions and their Applications to Physics, second edi., prepared by A. Gray and T. M. MacRobert, Macmillan, London, 1922.

[11] Y. L. Luke, Integrals of Bessel functions, McGraw-Hill, New York, 1962.

[12] F. W. J. Olver, D. W. Lozier, R. F. Boisvert and C. W. Clark (Editors), NIST Handbook of Mathematical Functions [With 1 CD-ROM (Windows, Macintosh and UNIX)], U. S. Department of Commerce, National Institute of Standards and 
Technology, Washington, D. C., 2010; Cambridge University Press, Cambridge, London and New York, 2010 (see also [1]).

[13] F. Oberhettinger, Tables of Mellin Transforms, Springer-Verlag, New York, 1974.

[14] A. D. Poularikas (Editor), Transforms and Applications Handbook, Second Edition, CRC Press, 2000.

[15] S. O. Rice, On contour integrals for the product of two Bessel functions, Quart. J. Math. Oxford Ser. 6 (1935), 52-64.

[16] H. M. Srivastava and J. Choi, Zeta and q-Zeta Functions and Associated Series and Integrals, Elsevier Science Publishers, Amsterdam, London and New York, 2012.

[17] H. M. Srivastava and M. C. Daoust, A note on the convergence of Kampé de Fériet's double hypergeometric series, Math. Nachr. 53 (1985), 151-159.

[18] H. M. Srivastava and H. Exton, A generalization of the Weber-Schafheitlin integral, J. Reine Angew. Math. 309 (1979), 1-6.

[19] H. M. Srivastava and P. W. Karlsson, Multiple Gaussian Hypergeometric Series, Halsted Press (Ellis Horwood Limited, Chichester), John Wiley and Sons, New York, Chichester, Brisbane and Toronto, 1985.

[20] Z. X. Wang and D. R. Guo, Special functions, Singapore:World Scientific; 1989.

[21] G. N. Watson, A Treatise on the Theory of Bessel Functions, Cambridge Mathematical Library edition, Camdridge University Press, 1995, Reprinted 1996.

[22] E. T. Whittaker and G. N. Watson, A Course of Modern Analysis: An Introduction to the General Theory of Infinite Processes and of Analytic Functions; With an Account of the Principal Transcendental Functions, Fourth edition, Cambridge University Press, Cambridge, London and New York, 1963.

Junesang Choi

Department of Mathematics, Dongguk University,

Gyeongju 780-714, Republic of Korea.

E-mail: junesang@mail.dongguk.ac.kr

Praveen Agarwal

Department of Mathematics,

Anand International College of Engineering,

Jaipur-303012, India.

E-mail: goyal.praveen2011@gmail.com 